\title{
Proximal, Microbiological and Color Evaluation and Comparison of the Meat of Llamas (Lama glama) and Alpacas (Vicugna pacos)
}

\section{Evaluación y Comparación Proximal, Microbi- ológica y del Color de la Carne de Llama (Lama Glama) y Alpaca (Vicugna Pacos)}

\section{Flores Mancheno, I. Salgado Tello, T. Sánchez Herrera}

I International Seminar of Livestock and Agroindustrial Production ESPOCH 2020

Corresponding Author:

C. Flores Mancheno

ifloresm1@yahoo.es

Published: 2 September 2021

Production and Hosting by

Knowledge E

(c) C. Flores Mancheno et al. This article is distributed under the terms of the Creative Commons Attribution License, which permits unrestricted use and redistribution provided that the original author and source are credited.
Facultad de Ciencias Pecuarias, Escuela Superior Politécnica de Chimborazo, Riobamba, Ecuador

\section{Abstract}

In this study, the meat of llama (Lama glama) and alpaca (Vicugna pacos) were examined. Data were analyzed through the analysis of variance (ANOVA) using the Centurion Statgraphics software. The bromatological characteristics of the meat were evaluated through proximal analysis. A high percentage of protein content was identified, with a higher value in the llama meat (24.025\%). Regarding the ethereal extract, minimum values were obtained in the alpaca meat $(2.707 \%)$, indicating lean meat. The humidity was variable depending on the conditions of the conservation, and the organic matter present in the meat defined the ash content, which was not higher than $5 \%$. In terms of the microbiological analyses, the value of viable aereobic mesophiles in Ilama meat was $1330 \mathrm{cfu} / \mathrm{g}$, and in alpaca meat it was $1490 \mathrm{cfu} / \mathrm{g}$; and the values for $E$. coli were $0.500 \mathrm{cfu} / \mathrm{g}$ and $1,250 \mathrm{cfu} / \mathrm{g}$, respectively.

Keywords: software, Lama glama, Vicugna pacos, proximal analysis, microbiological requirements.

\section{Resumen}

La carne de Llama (Lama glama) y Alpaca (Vicugna pacos) cumple con los requisitos para ser considerada como carne; Mediante el análisis de varianza (ANOVA) utilizando el Software Statgraphics Centurion se evalúa los requisitos bromatológicos por medio del análisis proximal de las carnes en estudio, donde se identifica un alto porcentaje en el contenido de proteína, lo que conlleva a un elevado valor biológico en ambas especies, siendo más destacada, en la carne de llama con 24,025\%; En cuanto a extracto etéreo se obtiene valores mínimos en especial en la carne de alpaca con $2,707 \%$, favoreciendo a la obtención de carne magra; En lo referente a humedad va a ser variable dependiendo de las condiciones de conservación de la misma, mientras tanto la materia orgánica presente en la carne define el contenido de cenizas, el cual no podrá ser mayor al 5\%. En relación a los análisis microbiológicos los valores de Mesófilos Aereobios Viables en la carne de llama fueron $1330 \mathrm{ufc/g}$, en la carne de alpaca $1490 \mathrm{ufc/g}$, para E. Coli de 0,500 ufc/g, y 1,250 ufc/g respectivamente encontrándose dentro de lo establecido en la Norma, y valores no permisibles por la presencia de Coliformes Totales.

Palabras Clave: software, Lama glama, Vicugna pacos, análisis proximal, requerimientos microbiológicos. 


\section{Introducción}

La Llama (Lama glama) y la Alpaca (Vicugna pacos) son camélidos que se crian de manera masiva en la zona andina de Perú, Bolivia, Argentina y Chile, en altitudes entre 2800 y 5000 metros sobre el nivel del mar [1], las cuáles representan un ingreso económico favorable para sus pobladores, especialmente campesinos de la zona, ya que su fibra y carne son aprovechables de manera industrial y artesanal. Los Camélidos Sudamericanos convierten, con inusual eficiencia, los pastos naturales de baja calidad nutricional en productos de alta calidad como son la fibra y la carne [2]. Ya que estas especies son adaptables a diversas condiciones nutricionales y estacionales, su alimentación es variada y se adapta al follaje o pasturas de valor nutricional de la época del año. De ésta manera se menciona que la carne de las dos especies presenta un gran valor nutricional.

Los carne de la llama (L. glama) y la alpaca (V. pacos) es considerada la fuente de proteína mas importante para la población, tanto por su valor nutricional como por su calidad. La llama tiene un gran potencial como fuente de carne en el antiplano, produciendo carcasas mas grandes que las de alpacas (L. pacos). Así mismo, el cuarto posterior presenta los cortes de mas valor y una mayor proporción de músculo que la alpaca [2], la alpaca es considerada de gran valor nutricional, por su bajo contenido de grasa y colesterol [3], en general la composición proximal de éstas dos especies en comparación a la carne de abasto generalmente se consume como carne de cerdo y de res, presenta características notablemente buenas con lo que respecta al contenido de proteínas.

La problemática encontrada en este tipo de carne son las malas condiciones de faenamiento que presentan, provocando que la carga microbiana aumente rápidamente después del la muerte del animal existen escasos mataderos que se destinen únicamente al sacrificio de camélidos, generalmente es un lugar en donde muchas especies son sacrificadas bajo condiciones poco controladas. Las condiciones higiénicas de los mataderos formales son generalmente aceptables y cuentan con servicios de inspección sanitaria. Por otro lado, el clandestino (fuera de los mataderos) se lleva a cabo en condiciones higiénicas poco adecuadas y carentes de control sanitario e inspección veterinaria [4]. Generalmente en la mayoría de países se utiliza los mataderos clandestinos y éste es el principal factor de contaminación microbiana en la carne de camélidos, es por bien decir que todos los beneficios que presenta el consumo de la carne de llama (L. glama) y la alpaca ( $V$. pacos) es perjudicado por el mal manejo del animal post-mortem que existe en los distintos lugares de expendio, ya que puede provocar diversas enfermedades microbianas o intoxicación a quienes lo consumen e incluso puede provocar la muerte. El mayor problema que limita la aceptación de la carne de camélidos para el consumo humano, es de la sarcocistiosis, enfermedades parasitarias que no afecta al hombre pero altera su aceptabilidad al generar un aspecto desagradable al producto, y ser confundido con otra parasitosis de alto potencial zoonótico [5]. El color de la carne también se ve influenciado por el faenamiento, pero depende fundamentalmente de los hemopigmentos: La hemoglobina y la mioglobina. La determinación de la calidad de la canal es uno de los factores más importantes 
desde el punto de vista económico, tanto para el productor como para la industria, puesto que está condicionada por las exigencias del mercado y tiene un efecto directo en el precio [6].

El presente artículo tiene como objetivo realizar la evaluación y comparación proximal, microbiológica y de color de la carne de llama (L. glama) y alpaca (V. pacos).

\section{Materiales y Métodos}

En este trabajo de investigación se empleó el software Statgraphics Centurión [7], proporcionando de este modo una explicación descriptiva de todas las variables durante el análisis proximal y microbiológico realizado a la carne de llama (L. glama) y la alpaca ( $V$. pacos), dichos análisis se realizaron utilizando gráficos que expliquen la respectiva distribución; para lo cual se empleó 4 muestras para cada tipo de carne, se efectuaron 3 réplicas de cada una, se procedió a realizar el análisis de varianza (ANOVA) utilizando el software mencionado, las muestras fueron consideradas como un factor bloque, mientras en factor de interés fue el tipo de carne (Alpaca, Llama). Las características generales de las muestras que se evaluaron provienen de especies de camélidos sudamericanos machos, de una edad aproximada de 18 meses de edad, la alimentación diaria se realizó bajo el sistema de pastoreo común en praderas nativas del tipo pajonal con predominancia de Stipa ichu y Festuca dolicophylla, las muestras fueron tomadas de las piernas de los animales en estudio, los mismos que fueron faenados o sacrificados de acuerdo a la metodología seguida para bovinos que comprende los siguientes pasos: (a) Aturdimiento, (b) Colgado, (c) Degüello, (d) Desangrado, (e) Desolle y (f) Eviscerado.

\section{Resultados y Discusión}

\subsection{Resultados análisis proximal}

\section{Table 1}

Resumen del análisis de varianza (ANOVA), análisis proximal (Flores et al., 2020).

\begin{tabular}{|l|l|l|} 
& Carne & Media \\
\hline Humedad [\%] & Alpaca & 74,363 \\
\hline Lenizas [\%] & Alama & 73,300 \\
\hline Proteína [\%] & Llama & 1,558 \\
\hline EE [\%] & Alpaca & 23,338 \\
\hline & Llama & 24,025 \\
\hline & Alpaca & 2,707 \\
\hline
\end{tabular}

Desviación
Estándar
0,670
1,178
0,237
0,154
2,364
0,575
0,430
0,658

\begin{tabular}{|l|l|}
\hline P-valor & Diferencia \\
\hline $\mathbf{0 . 0 1 3}$ & 1,063 \\
\hline $\mathbf{0 . 0 1 3}$ & 0,220 \\
\hline 0.284 & $-0,786$ \\
\hline 0.078 & $-0,443$ \\
\hline
\end{tabular}

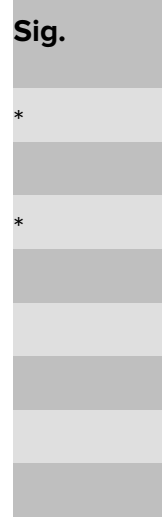

${ }^{*} P$-valor $<0,05$ indica diferencias significativas. 
Según indica [8] los análisis comprendidos dentro de este grupo, también conocido como análisis proximales Weende, se aplican en primer lugar a los materiales que se usarán para formular una dieta como fuente de proteína o de energía y a los alimentos terminados, como un control para verificar que cumplan con las especificaciones o requerimientos establecidos durante la formulación. Por lo que los resultados que se arrojan en esta investigación tendrán mucha valía al momento de tomar decisiones sobre la importancia del consumo directo al igual que para su posible uso en la industria de los derivados cárnicos. Observando en la Tabla 1, se puede concluir que la Humedad y Cenizas muestran diferencias significativas entre los diferentes tipos de carne con diferencias de $1,063 \%$ y $0,220 \%$ respectivamente, teniendo en cuenta como lo indica [9] que poder determinar el contenido de humedad de un alimento rápidamente puede optimizar de manera significativa un proceso de fabricación, consiguiendo la misma influir en gran medida la fluidez de un material, compresibilidad, y cohesividad.

Mientras que de la proteína y del Extracto Etéreo (EE) dada la evidencia, no se puede asumir que exista tal diferencia, conociendo que las carnes más magras contienen más proteína y menos grasa, y debido a que el agua es un componente de la proteína (pero no de grasa), un corte más magro va a contener un poco más de agua en base a peso [10].

\subsection{Humedad}

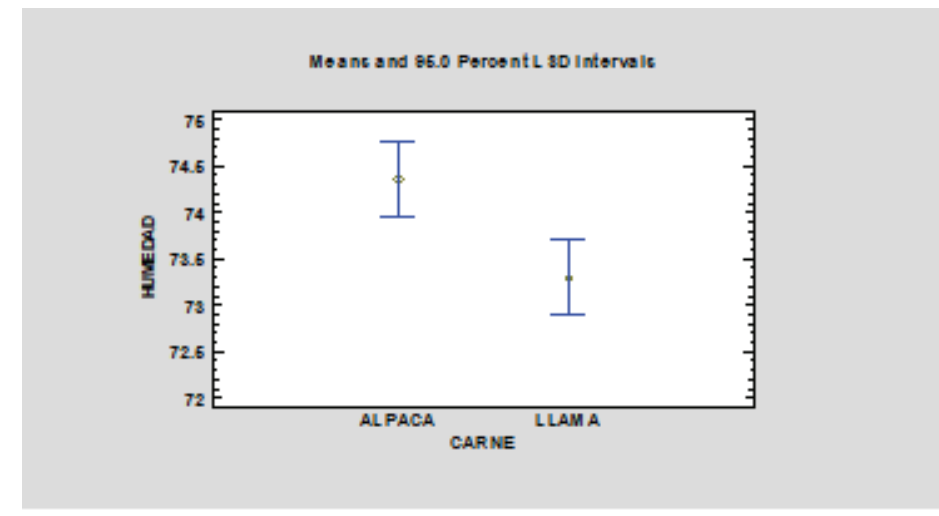

\section{Figure 1}

Análisis proximal de la humedad (Flores et al., 2020).

El análisis del contenido de humedad o de materia seca, es el análisis bromatológico que permite conocer el grado de dilución de los nutrimentos o componentes de la muestra como lo indica [11]. Al presentar los resultados referentes a Humedad en la Figura 1, mediante las medias determinadas de cada una de las especies de camélidos sudamericanos en estudio, presentaron diferencias significativas de 1,063\% entre especies, con un valor de $74,363 \%$ para la Alpaca y $73,300 \%$ en la Llama, esta diferencia puede estar correlacionada a que el análisis se realizó en fresco para la Alpaca y con una carne conservada para Llama, y como indica a temperaturas bajas de conservación las células del producto se 'sueltan' un poco y parte de la humedad 
se derrite y escurre gradualmente. De acuerdo al valor de humedad reportado por [12] obtuvo el $(73,90 \%)$ en carne de llama con acción de refrigeración y $(74,10 \%)$ en la de alpaca en condiciones normales, dicha variación involucra al factor de refrigeración ya que es susceptible a perdida de humedad debido a la escaza presencia de grasa subcutánea en la conformación de las canales lo cual provoca perdida por goteo al estar expuesta a una refrigeración previa.

\subsection{Proteína}

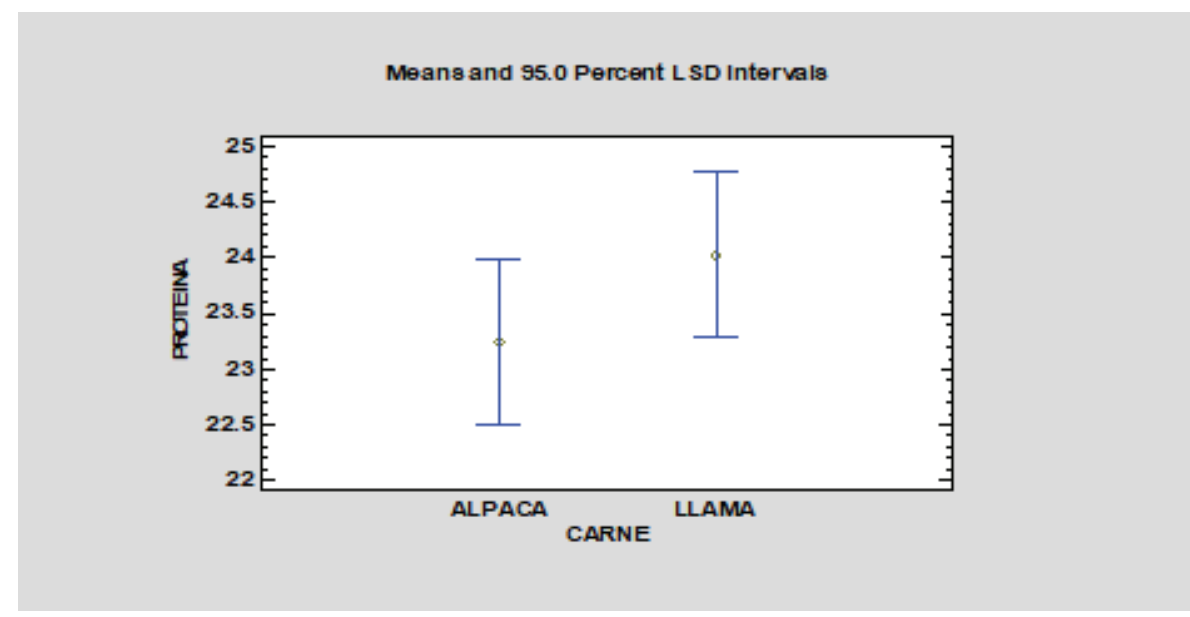

Figure 2

Análisis proximal de la proteína (Flores et al., 2020).

La presencia de proteína en la carne es importante ya que es aprovechado por el organismo humano, en un ( $97 \%$ de las proteínas y $96 \%$ de las grasas). De igual manera ocurre con el consumo de vísceras, como los riñones, hígado y corazón, puesto que son aprovechadas por el organismo no solo por el contenido mineral y vitamínico, sino también por su contenido de proteínas de alta calidad [13]. Los resultados expuestos en la Figura 2 correspondiente a la proteína indica que no presenta diferencias significativas $(p<0,05)$, entre especies, teniendo $23,239 \%$ para Alpaca y $24,025 \%$ para la Llama, siendo un contenido mayor a lo que presenta el resto de especies animales que proporcionan proteína mediante el consumo de carne. Se mantiene una tendencia similar con los datos reportados por [1] que obtuvo un contenido protéico de $(23,9 \%)$ en llama y un valor de $(22,7 \%)$ en alpaca, al relacionar estos datos con los de la norma [14] se determina que los datos de la presente investigación excede el contenido de proteína mínimo establecido por la norma que debe ser del 12\%, lo cual implica mayor valor biológico en cuanto aminoácidos esenciales. Siendo un valor muy considerable para los procesos de nutrición humana ya que como menciona [15] la digestibilidad verdadera se refiere, las proteínas de origen animal (huevos, leche, pescado y carne) presentan valores en torno al 95\%, mientras que las de naturaleza vegetal comúnmente incluidas en nuestras dietas, son netamente inferiores. 


\subsection{Cenizas}

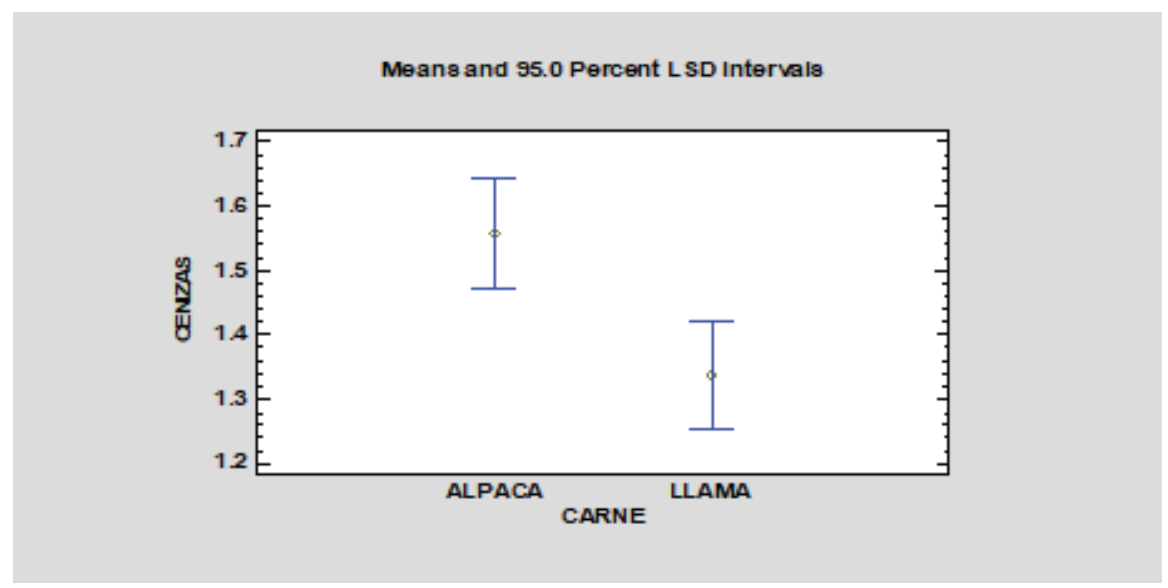

Figure 3

Análisis proximal de cenizas (Flores et al., 2020).

La determinación de cenizas como lo señala [16] es referida como el análisis de residuos inorgánicos que quedan después de la ignición u oxidación completa de la materia orgánica de un alimento, por lo que es importante conocer cuál es el contenido inorgánico que poseen los tipos de carnes y que se encuentra reportados en la Figura 3, indicando una diferencia significativa de 0,220\% entre los tipos de carne, de igual manera se puede manifestar que se localiza en correlación con la diferencia de humedad que se obtuvo en la investigación, ya que el proceso de obtención de cenizas en el alimento dependerá del contenido de humedad en relación al tiempo de cocción. Aun así, según lo establecido en [14] determina que el contenido de cenizas máximo debe ser del 5\%; Conforme a los porcentajes resultantes entre especies, teniendo 1,34\% para Alpaca y $1,56 \%$ para la Llama, por lo cual se precisa que los valores obtenidos están dentro del rango admisible establecido por la norma; De acuerdo a [1] los valores correspondientes a cenizas son de $(1,2 \%)$ en la carne de llama mientras que para la carne de alpaca es de (1,1\%), lo cual reafirma que los porcentajes obtenidos y registrados son valores con cierta similitud, sin embargo varían debido a la materia orgánica que contienen la carne de cada animal.

\subsection{Extracto etéreo}

Sin existir diferencias entre el contenido del extracto etéreo para los camélidos sudamericanos en estudio, siendo 2,207\% para Alpaca y 2,928\% para Llama, se puede determinar que el contenido de materiales liposolubles presentes es mínimo, siendo un producto casi magro. Mientras que según [17] el porcentaje de grasa bruta o extracto etéreo de la carne de Llama es de (2,1\%), en tanto que el valor registrado por la carne de alpaca corresponde al (1,16\%); Por lo tanto, se comprueba que dichos porcentajes se encuentran dentro de los citados por [14] que determina que el contenido de extracto etéreo máximo debe ser del $20 \%$; Por lo cual los valores presentes en la 


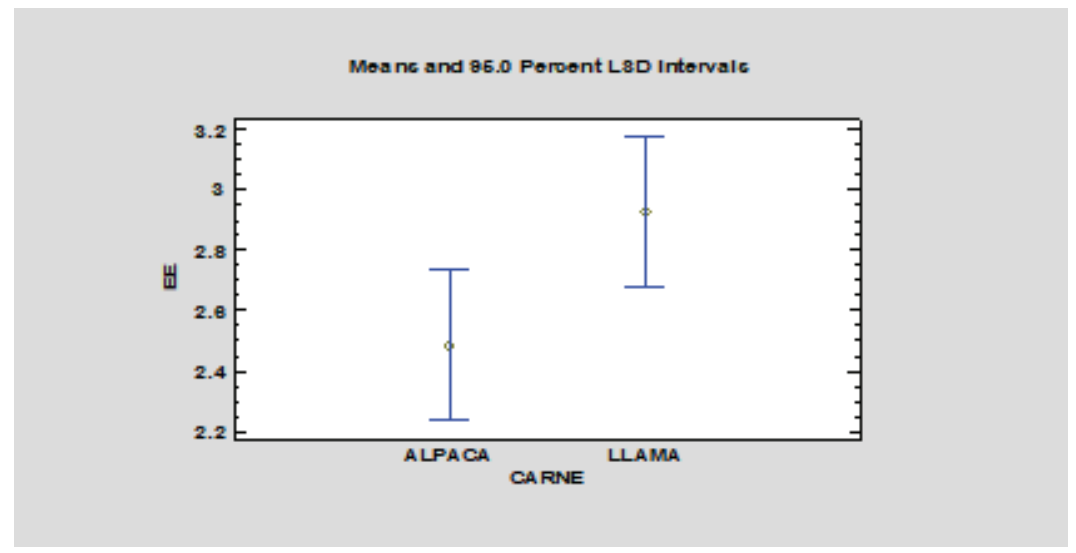

\section{Figure 4}

Análisis proximal del extracto etéreo (Flores et al., 2020).

carne de los camélidos estudiados se consideran aptos para incluirse en una dieta equilibrada y que ayudan a presentar a la carne de una calidad y terneza ideal como lo indica [18] que menciona que en gran medida la terneza de la carne, además de factores como el contenido de grasa subcutánea o intramuscular (particularmente en canales con limitada cantidad de grasa subcutánea) y su relación directa con la tasa de enfriamiento post mortem, consecuente incremento de la actividad autolítica a nivel muscular y paralela disminución del acortamiento miofibrilar, está explicada por la cantidad y tipo de tejido conjuntivo presente en la carne. En los datos obtenidos influye directamente que las muestras se obtuvieron de animales relativamente jóvenes los cuales se mantienen por las noches en un corral dormidero y la alimentación diaria es en praderas nativas.

\subsection{Análisis microbiológico}

\section{Table 2}

Resumen del análisis de varianza (ANOVA), análisis microbiológico (Flores et al., 2020).

\begin{tabular}{|c|c|c|c|c|c|c|}
\hline & Carne & $\begin{array}{l}\text { Media } \\
\text { [ufc/g] }\end{array}$ & $\begin{array}{l}\text { Desviación } \\
\text { estándar } \\
{[\text { ufc/g] }}\end{array}$ & P-valor & Diferencia & Sig. \\
\hline \multirow[t]{2}{*}{ Mesófilos Aerobios Viables } & Alpaca & 1497,500 & 221,679 & 0,006 & 160,000 & $*$ \\
\hline & Llama & 1337,500 & 219,184 & & & \\
\hline \multirow[t]{2}{*}{ Coliformes Totales } & Alpaca & 183,750 & 46,256 & 0,110 & 39,000 & \\
\hline & Llama & 144,750 & 53,075 & & & \\
\hline \multirow[t]{2}{*}{ E-Coli } & Alpaca & 1,250 & 1,500 & 0,215 & 0,750 & \\
\hline & Llama & 0,500 & 1,000 & & & \\
\hline
\end{tabular}

${ }^{*} P$-valor $<0,05$ indica diferencias significativas.

Las enfermedades de transmisión alimentaria (ETA) constituyen uno de los principales problemas de Salud Pública en el mundo. La incidencia de éstas se relaciona 
con deficiencias higiénico-sanitarias de los alimentos durante su procesamiento, o por el uso de materia prima contaminada [19], por lo que los productos cárnicos de origen vacuno pueden contaminarse en cualquiera de las etapas de procesamiento, ya que este tipo de ganado es un reservorio natural de microbiota intestinal y patógenos para el humano, por lo que sus heces son fuente significativa de microorganismos [20].

Siendo importante el análisis microbiológico de los microrganismos indicadores del proceso de faenamiento y posterior maduración que se llevó a cabo en los dos tipos de carne, para conocer cuál es la calidad sanitaria y si nos encontramos dentro de las normas establecidas por el país.

\subsection{Aerobios mesófilos}

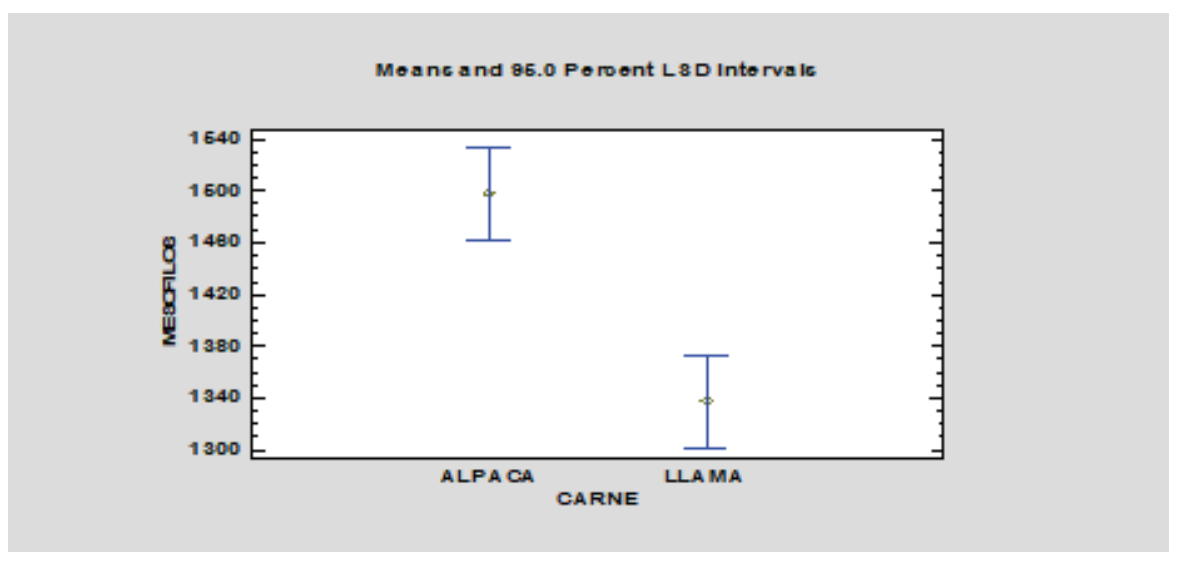

Figure 5

Análisis microbiológico, mesófilos (Flores et al., 2020).

Observando los resultados se puede apreciar una diferencia significativa en la cantidad de Mesófilos Aerobios viables identificando en la carne de llama 1330 ufc/g, mientras que en la carne de alpaca se presentó un incremento, alcanzando $1490 \mathrm{ufc/g}$, dichos valores conllevan una diferencia de $160 \mathrm{ufc} / \mathrm{g}$, esto puede deberse al proceso de faenamiento que se llevó a cabo en diferentes días y las condiciones no fueron las mismas para las dos especies, permitiendo que exista un incremento microbiano en indicadores de presencia de ciertos microorganismos que se encuentran en el ambiente.

A pesar de ello se encuentran dentro de los valores permisibles según establecido en [14] que determina que el nivel de aceptación mínima debe ser de 1,0×10^6 Mesófilos Aerobios viables en productos cárnicos crudos.

\subsection{Coliformes fecales}

Los resultados en cuanto a Coliformes Fecales no presentan diferencias entre sí, es decir sus valores son semejantes, ya que la carne de llama presentó 144,750 ufc/g, mientras que en la carne de alpaca, hubo un incremento poco perceptible, alcanzando 


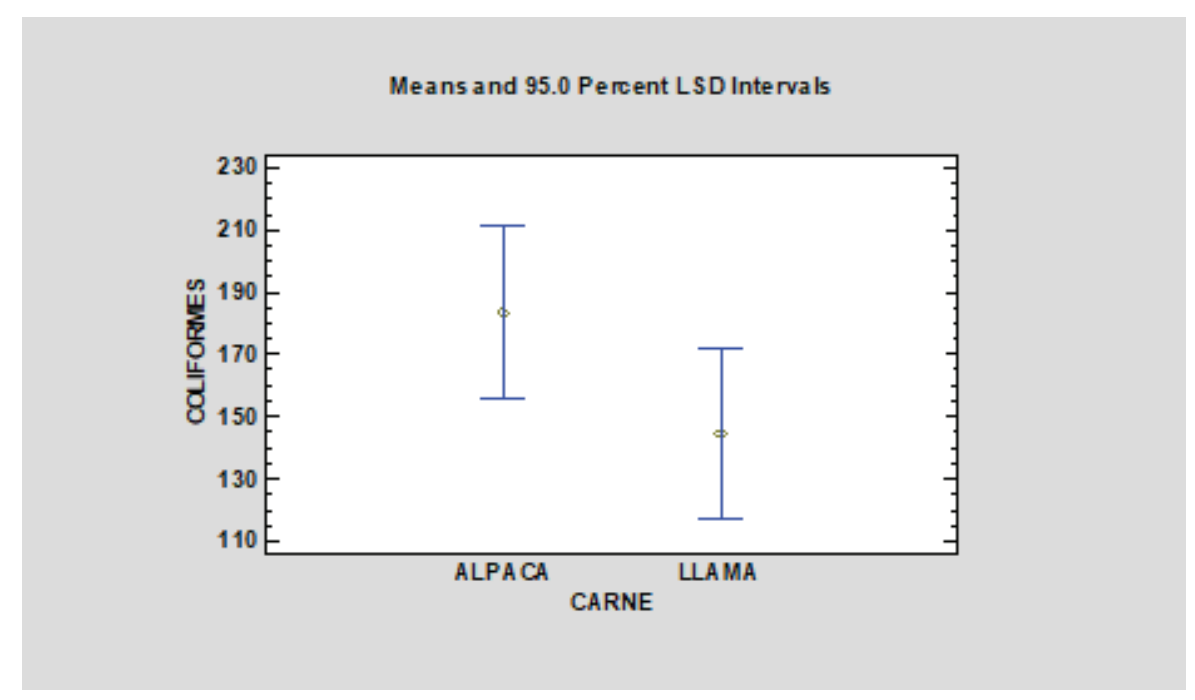

Figure 6

Análisis microbiológico, coliformes (Flores et al., 2020).

$750 \mathrm{ufc} / \mathrm{g}$, dichos valores determinan una disimilitud de $0,110 \mathrm{ufc} / \mathrm{g}$, valor que sirve como indicador de contaminación, debido a que la presencia de este tipo de bacterias en los alimentos denota que ya no son aptos para el consumo humano ya que muestra indicios de contaminación fecal ya sea por el faenamiento, agua, contaminación cruzada o por contacto humano directo durante la manipulación de la carne [21]; Todos los animales transportan grandes cantidades de microorganismos. Numerosas bacterias, además de mohos y levaduras, están presentes en el cuero, los pelos y las pezuñas de los vacunos, y son transmitidos a la carcasa luego del sacrificio. Los restos de estiércol suelen acceder al músculo, así como el contenido intestinal si la evisceración no se hace cuidadosamente. Por otra parte, las bacterias también pueden proceder de los pisos, paredes, mesadas, cuchillos y manos de los operadores en la planta de faena [22]; Por tal motivo según [14] es inaceptable la presencia de coliformes fecales.

\subsection{Escherichia coli}

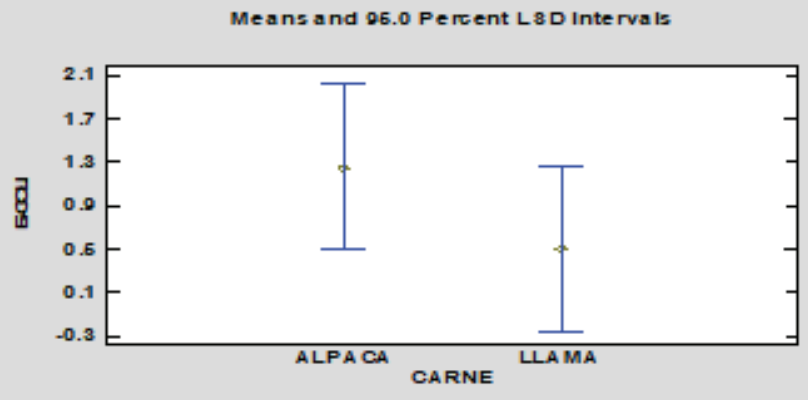

Figure 7

Análisis microbiológico, E. Coli (Flores et al., 2020). 
Al obtener los resultados se puede percibir que no existe diferencia significativa en la cantidad de $E$. coli, debido a que en la carne de llama se observó 0,500 ufc/g, mientras que en la carne de alpaca se obtuvo un valor de 1,250 ufc/g, dichos valores conllevan a una diferencia de $0,750 \mathrm{ufc} / \mathrm{g}$, esto puede deberse a la temperatura expuesta a la carne posterior al faenamiento, actividad de agua en la carne que difieren en cada una de las especies así como también el no alcanzar pH óptimo de la carne, lo cual permite que exista un incremento microbiano en indicadores de presencia de ciertos microorganismos que se encuentran en el ambiente; Sin embargo según [14] que determina el nivel aceptable de ufc/g de $E$. coli, establece que los valores obtenidos son admisibles, debido a que no superan el nivel máximo $1,0 \times 10 \wedge 3 \mathrm{ufc} / \mathrm{g}$ de $E$. coli detallado en la norma, que indican una baja presencia del microorganismo, de ahí la importancia de la detección oportuna y precisa de este microorganismo, ya que generalmente se encuentra en bajas concentraciones, son fácilmente superados por la flora de competencia, su aislamiento toma mucho tiempo y depende de la sensibilidad de los medios y técnicas utilizados [23].

\subsection{Análisis del color}

Table 3

Resumen del análisis de varianza (ANOVA) (Flores et al., 2020).

\begin{tabular}{|c|c|c|c|c|c|c|}
\hline & Carne & Media & $\begin{array}{l}\text { Desviación } \\
\text { Estándar }\end{array}$ & P-valor & Diferencia & Sig. \\
\hline \multirow[t]{2}{*}{$\mathbf{L}$} & Alpaca & 35,690 & 0,760 & 0,334 & 1,993 & \\
\hline & Llama & 33,690 & 3,200 & & & \\
\hline \multirow[t]{2}{*}{ a } & Alpaca & 14,970 & 0,590 & 0,320 & 1,253 & \\
\hline & Llama & 13,720 & 1,610 & & & \\
\hline \multirow[t]{2}{*}{ b } & Alpaca & 1,317 & 0,120 & $-5,647$ & 0,750 & $*$ \\
\hline & Llama & 6,965 & 2,189 & & & \\
\hline
\end{tabular}

Existe poca o nula investigación colorimétrica en lo que corresponde al color de las carnes provenientes de la Llama y Alpaca por lo que la discusión se centrará en los resultados obtenidos por los investigadores que indican en la Tabla 1, que nos presentan los diferentes componentes que son parte de la evaluación colorimétrica utilizada mediante el modelo CIELAB, en donde $\mathrm{L}^{*}$ corresponde a la luminosidad del color, a* es el croma donde los valores positivos corresponden a rojo y los negativos al color verde, $b^{*}$ es el tono para este componente los valores positivos corresponden a amarillo y aquellos valores negativos se refieren al color azul. Por lo que se puede mencionar que el valor de $b^{*}$ es el único que tiene una diferencia significativa entre los dos tipos de carne con una diferencia de $-5,647$, esto puede deberse al tipo de alimentación que tiene cada una de las especies ya que la llama por el entorno en el que vive ésta se alimenta en su mayoría a través del pastoreo el cual hace que las grasas sean de tonalidades mucho más amarillas. 
Por otra parte, la mioglobina que contienen los dos tipos de carne y que son los responsables de la coloración de los mismos, estuvieron expuestos a cambios ya que en el proceso de faenamiento, muestreo y posterior análisis colorimétrico se encontraron en contacto directo con el oxígeno, generando modificaciones químicas (cambios redox) que cambiaron la refracción de la luz, de igual manera las concentraciones de mioglobina varían de especie animal, ya sea por el tipo de músculo (actividad muscular), raza y edad.

\section{Conclusiones}

Los resultados obtenidos en el presente trabajo se pueden resumir en las siguientes conclusiones:

- La carne de la llama (L. glama) y la alpaca (V. pacos) presentan valores similares en proteinas y estracto etereo o grasa bruta, pero con diferencias significativas en humedad y cenizas. Estos valores comparados a la carne de abasto, presentan mayor contenido de humedad, proteinas y grasas, pero un valor similar en el contenido de cenizas en comparacion a los otros animales.

- El análisis microbiológico presentó resultados poco favorables, con valores permisibles para Mesófilos Aereobios Viables y E. coli en la carne de alpaca y llama pero con presencia de Coliformes Totales no permisibles tanto en la carne de llama y alpaca. Siendo estas bacterias las causantes de varias enfermedades de intoxicación en la población andina.

- El color de la carne tanto de la llama y alpaca evaluado mediante el modelo CIELAB, permite ver que la carne presenta una luminosidad al color (L) y croma (a) similares, mientras que los tonos (b) presentan diferencias significativas, es decir los dos tipos de carne presentan un color rojo pero la llama presenta una coloración mas amarilla con respecto a la grasa, aumentando el indice de amarillo y rojo con el tiempo de maduración de la carne.

\section{References}

[1] Mamani L, Cayo F, Gallo C. Caracteristicas de canal, calidad de carne y composición química de carne de llama: Una revisión. Scielo Perú. 2014.

[2] Robles R. Respuesta productiva y rendimiento de carcasa de llamas (Lama glama) dientes de leche sometidas a engorde, cuatro tipos de alimentación [Tesis para el grado de Magister Scientiae en nutrición]. Lima; 2018.

[3] Medina-Valdiviezo K. Evaluacion de las propiedades tecnologicas de la carne de Alpaca (Vicugna pacos) envasada al vacio durante el almacenamiento en congelacion. Lima; 2014.

[4] Carpio-Cuadra G. Propiedades tecnológicas de la carne de llama (Lama glama) marinada con cloruro sódico y fosfatos sometidas a congelación y descongelación. Lima: 2006.

[5] FAO. Ecuador; [4-7pg.]. Disponible en: http://www.fao.org/fileadmin/user_upload/agns/pdf/Preventing_ Ecoli_es.pdf

[6] Franco J. Importancia de los factores productivos, tecnológicos y de manejo en la calidad de la canal y de la carne vacuna. En: Bianchi G, Feed O (ed). Introducción a la ciencia de la carne. Buenos Aires: Hemisferio Sur; 2009.

[7] Batanero C. Análisis de datos con statgraphics. San Jorge-Granada; 2008.

[8] FAO. Ecuador: FAO; 2009. Disponible en: http://www.fao.org/3/AB489S/AB489S03.htm 
[9] AOAC. Métodos de análisis de la asociación oficial de química analítica para determinar humedad, fibra, cenizas, grasa y proteína. Washington: AOAC; 2003.

[10] USDA. Ecuador: USDA; 2009. Disponible en: https://www.fsis.usda.gov/wps/wcm/connect/ Od924688-b15d-490e-87ba-fad5b9d87727/Water_in_Meat_Poultry_SP.pdf?MOD=AJPERES

[11] Bradley R. Food analysis. Nielsen S, editor. New York: Kluwer Academic; 2003. Moisture and total solids analysis.

[12] Cristofanelli A. Características de canal, calidad de carne y composición química de carne de llama. Lima: Scielo; 2005.

[13] Fernández FP. Calidad de carne de vacuno: Concepto en España. Mundo Ganadero. 1991:5(marzo):3539.

[14] NTE INEN 1338. Carne y productos cárnicos. Salchichas. 1996. Disponible: http://repositorio.utn.edu. ec/bitstream/123456789/149/4/03\{\%\}20AGP\{\%\}2063\{\%\}20NTE\{\%\}2OINEN\{\%\}201338.pdf

[15] WHO. Energy and and Protein Requirements. Report of a Join FAO/WHO/ONU Expert Consultation. Technical Report Series 724. Ginebra; WHO: 1985.

[16] Ronald SK, Ronald S, Harol E. Composición y Análisis de los Alimentos de Pearson. Editorial Continental.

[17] Tenemaza T. Aplicaciones gastronómicas a base de carne de llama en el restaurante sumak kausay parroquia calpi 2010. Riobamba: Escuela Superior Politecnica de Chimborazo; 2010.

[18] González-Flores T, Rojas-Herrera RA. Enfermedades transmitidas por alimentos y PCR: Prevención y diagnóstico. Salud Pub Mex. 2005;47:388-390.

[19] Gallegos M, Morales A, Álvarez G, Vásquez J, Morales L, Martínez I, Maldonado J. Caracterización de aislados de Escherichia coli O157:H7 en canales de bovinos y porcinos mediante PCR. Rev Cient FCV-LUZ. 2009;2:139-146.

[20] Bianchi G, Feed O. Calidad de la carne y de productos cárnicos ovinos introducción a la ciencia de la carne. Montevideo:

[21] FAO. Ecuador; 2005. Disponible en: http://www.fao.org/tempref/GI/Reserved/FTP_FaoRIc/old/prior/ segalim/animal/paises/pdf/2914ecu.pdf

[22] ICMSF. Microorganisms in foods - Microbial ecology of food commodities. (Vol. 6). London: Blackie Academic \& Professional; 1998.

[23] Perry JD, Freydiére AM. The application of chromogenic media in clinical microbiology. J Appl Microbiol. 2007;103:2046-2055. 